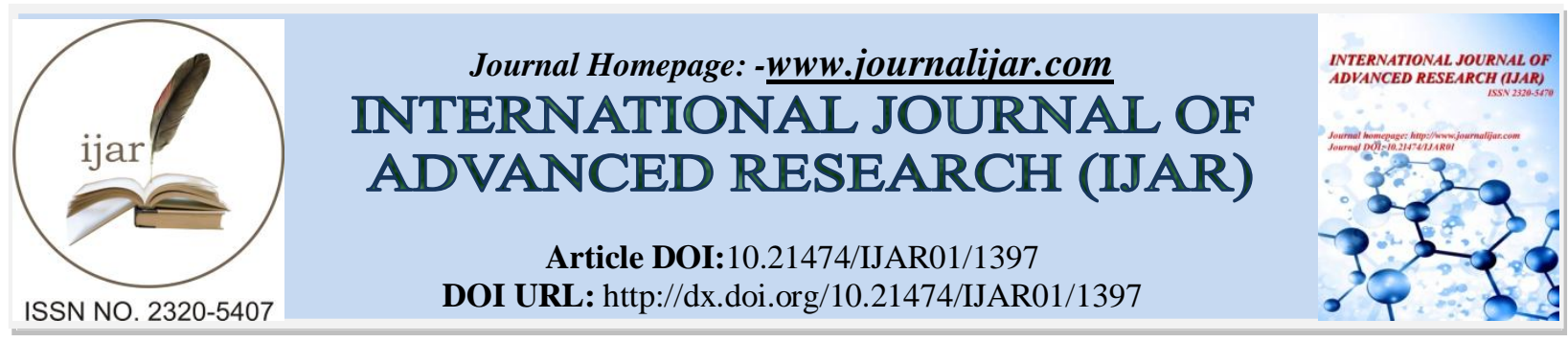

RESEARCH ARTICLE

\title{
DATA TRANSMISSION USING VISIBLELIGHT COMMUNICATION.
}

\author{
Adarsha $\mathbf{R}$ Dixit ${ }^{1}$, Adithya $\mathbf{S}^{\mathbf{1}}$, Anirudh $\mathbf{R}^{1}$, Naren $\mathbf{S}^{\mathbf{1}}$ and Lakshmi Bhaskar ${ }^{2}$. \\ 1. B.E. students, Dept. of TCE, BNMIT, Bangalore, India. \\ 2. Asst. Professor, Dept. of ECE, BNMIT, Bangalore, India.
}

\section{Manuscript Info}

Manuscript History

Received: 18 June 2016

Final Accepted: 13 July 2016

Published: August 2016

Key words:-

Visible Light Communication (VLC), ON-OFFKeying (OOK), Wireless Communication

\begin{abstract}
Due to the enormous traffic in the radio frequency spectrum, there is a need for an alternative means for wireless communication which can accommodate increasing traffic demand. Visible light communication (VLC) is one such alternative which can provide high information transmission, using light from LEDs as the communication medium. In these systems, light-emitting diodes blinks when information is transmitted through them, a sensitive photovoltaic-cell can detect the on-off behavior and decode the information embedded in it.

In this project analyses is done on various issues with current wireless communication systems, and discuss how visible light communications can resolve these issues. Later, the implementation process of the visible light communication system along with its design is described in detail. Thus achieved results of the system, in terms of transmission distance and type of data is discussed.
\end{abstract}

Copy Right, IJAR, 2016,. All rights reserved.

\section{Introduction:-}

In the coming years Visible light communication is a viable technology to accommodate the need for faster and better wireless communications. The basic idea, is that instead of using traditional methods of communication over cables or radio frequencies, VLC systems send data by turning light on (logic 1) and off (logic 0). This is the simplest method for digital communication using visual light. Any light technically could be used to transfer data but what matters the most is the brightness and the frequency of the light at which it modulates. The data rate of the transmission will depend on how speed at which the lights can turn on and off. LEDs are a popular choice for VLC communication because of their rapid on-off nature. There is one issue with fluorescent lights however. While they could be used for communications, they can only do so at relatively low frequencies, due to the fact that fluorescent bulbs cannot be turned on and off at high speed. The resulting transmission rate would be approximately $10 \mathrm{kbps}$. This rate is not high enough to support the transmission of data such as video or audio, while LEDs, have a much faster switching speed, as they are capable of providing up to $500 \mathrm{Mbps}$ and possibly even more

\section{Components:-}

Light Emitting Diode (LED)

The work here is concentrated on the semiconductors formed by P-type and N-type materials. Light emitting diode (LED) works on the principle of electroluminescence where PN junction semiconductor that emits light when forward biased releases the energy in the form of photons. What makes LED different from other diodes is the bandgap of the LED which is designed for radiative recombination. 

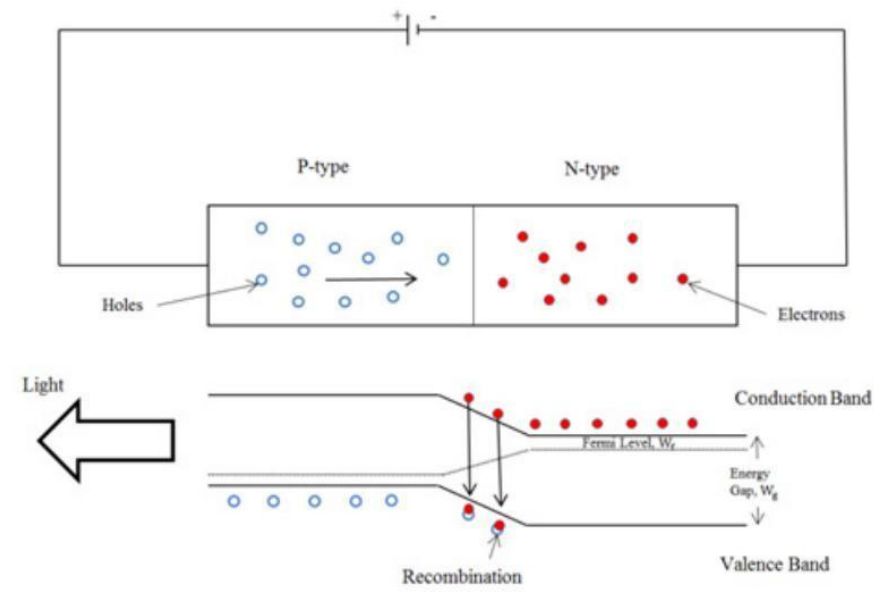

Fig. 2.3:- LED PN- junction biasing arrangement and the energy bands associated with the diode

When joining the N-type and P-type materials, the Fermi levels $\left(\mathrm{W}_{\mathrm{f}}\right)$ will be aligned and will produce an energy barrier even when there is no external voltage applied. There are two energy bands, namely the conduction band and valence band, which are separated by a forbidden region with the width of Wg. In the conduction band, electrons not bound to individual atoms are free to move. In the valence band, unbound holes are mobile and have positive charge. The free electrons in the $\mathrm{N}$ region cannot go up the barrier without theexternal energy; that is the same for the holes that cannot surmount the barrier. By applying a sufficient energy $(\mathrm{eV})$, a free electron crosses the barrier, falls into the lower energy level and recombines with the hole, releasing the energy in the form of photons.

\section{White Light Emitting Diodes:-}

Although every color can be produced by LEDs within the visible region, white light is the most desirable color for general illumination. The visible radiation detectable to the human eye is between $480 \mathrm{~nm}$ to $750 \mathrm{~nm}$. White light emission from an LED is through mixture of multi-color LEDs or by the combination of phosphors with blue/UV LED emission. There are different types of white LEDs. Some of the important ones are:

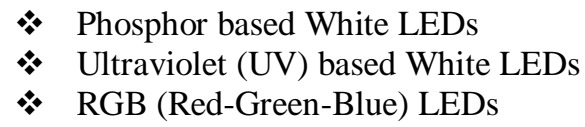

* Phosphor based White LEDs

The InGaN blue LED is coated with phosphor. The wavelength converting phosphors is combined with a blue LED to emit white light. The chip inside the LED emits blue light when external voltage is applied. The emitted blue light passes through the yellow phosphor, yielding white light emission.

\section{* Ultraviolet (UV) based White LEDs}

Ultraviolet LEDs were fabricated with pre-coating blue/green/red phosphors onto ultraviolet (UV) LED to emit white light.

\section{$*$ RGB (Red-Green-Blue) LEDs}

An RGB (red, green, blue) 3-chip LED is a mixture combination of three colors to produce white light with little variance in the Kelvin color temperature. What we see coming from the sun is white light. We know that the visible spectrum of radiation that the sun emits is actually a broad range of wavelengths, ranging from red to orange, yellow, green, and blue, indigo to violet (ROYGBIV). When this broad range of colors impinges on our retina, our brain interprets it as "white". A tri-color LED tries to mimic this effect by outputting a board range of wavelengths (red, green and blue). Note that the three dominant wavelengths of the tri-color LED are at the ends and the center of the visible spectrum, thus attempting to replicate the coverage of the range and getting close to (ROYGBIV) as possible (with minimal hardware). So, it is less of a mixing and more of an attempt to create a continuous function by using a few sampled points. 
The LED chosen for our project was a generic White LED with unknown specifications as an LED meeting our requirements was unavailable.

\section{LIGHT SENSING DEVICES:-}

o Photodiode

A photodiode converts the incident light into the current. It works on the principle called Photo-conduction, whereas LED works on the principle of electro-luminance. The photodiode is a type of photo detector which converts the light to either current or voltage.

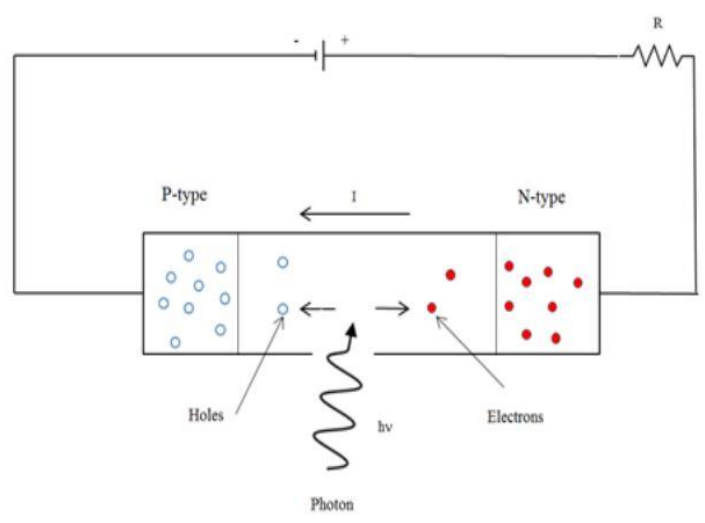

Fig. 2.2:- Basic biasing arrangement and construction of photodiode

A photodiode converts the incident light into the current. It works on the principle called Photo-conduction, whereas LED works on the principle of electro-luminance. The photodiode is a type of photo detector which converts the light to either current or voltage.

A photodiode has two modes of operation:

* Photovoltaic Mode

Photovoltaic mode, also called as zero bias operation, occurs when no external voltage is given to the photodiode. The photo-current generated is fixed and also linearly dependent on the incident radiation level.

\section{* Photoconductive Mode}

The diode is reverse biased (cathode positive and anode negative), which increases the depletion region width, reducing the junction capacitance. This results in faster response time. However, in this mode the effects of noise and dark currents will be more.

oPhotovoltaic Cell

A solar cell or photovoltaic cell is an electrical device that converts the energy of light directly into electricity by the photovoltaic effect, which is a physical and chemical phenomenon. It is a form of photoelectric cell, defined as a device whose electrical characteristics, such as current, voltage, or resistance, vary when exposed to light. Solar cells are the building blocks of photovoltaic modules, otherwise known as solar panels. 


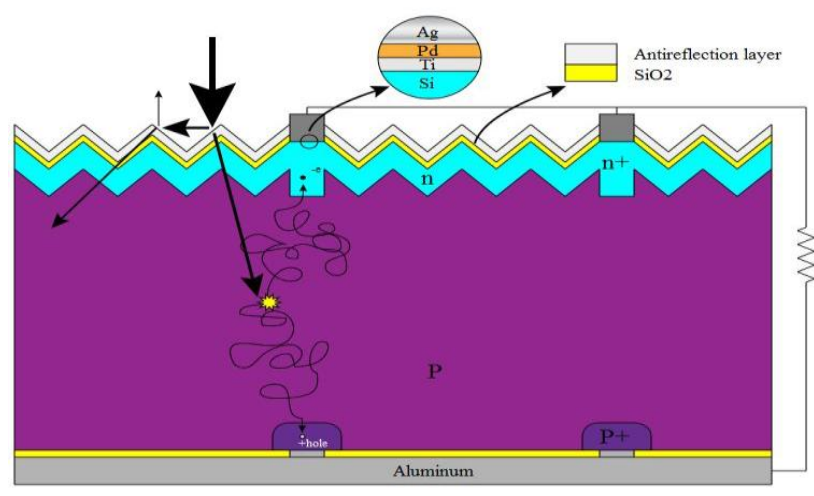

Fig. 2.3:- Arrangement in a Photovoltaic cell

A solar cell or photovoltaic cell is an electrical device that converts the energy of light directly into electricity by the photovoltaic effect, which is a physical and chemical phenomenon. It is a form of photoelectric cell, defined as a device whose electrical characteristics, such as current, voltage, or resistance, vary when exposed to light. Solar cells are the building blocks of photovoltaic modules, otherwise known as solar panels.

The solar cell works in several steps:

Photons in sunlight hit the solar panel and are absorbed by semiconducting materials, such as silicon.

Electrons are excited from their current molecular/atomic orbital. Once excited an electron can either dissipate the energy as heat and return to its orbital or travel through the cell until it reaches an electrode. Current flows through the material to cancel the potential and this electricity is captured. The chemical bonds of the material are vital for this process to work, and usually silicon is used in two layers, one layer being bonded with boron, the other phosphorus. These layers have different chemical electric charges and subsequently both drive and direct the current of electrons.

An array of solar cells converts solar energy into a usable amount of direct current (DC) electricity. The most commonly known solar cell is configured as a large-area $\mathrm{p}-\mathrm{n}$ junction made from silicon.

Our choice is the Photovoltaic cell due to its large surface area, fast response times and high sensitivity.

\section{Literature:-}

Nguyen, "Visible Light Communication", WPI, 2015 - In this paper the authors conducted a study and performed experiments on visible light communication. They concluded that visible light communication is a viable technology to accommodate the need for faster and better wireless communications in the coming years. The basic idea is that instead of using traditional methods of communication over cables or radio frequencies, VLC systems send data by turning light on (logic 1 ) and off (logic 0 ). Their report describes and evaluates the visible light communication system design they created. As visible light communication technology is relatively new, their team worked on creating a prototype to test out this technology and demonstrate its possible capabilities. Using an older system's design as a foundation, the team developed a successor with improved transmission specifications. The first part of the process is preparing a file or string of bytes for transmission. In order to synchronize the transmitter and receiver, the system divides the data into units called "frames", each starting with a preamble to let the receiver know that a transmission has started. The transmitter takes a file, breaks into frames, and inserts preamble sequences before each frame. Then it sends the modified file to a microcontroller unit (MCU) over the serial port. The MCU controls the gate of a transistor based on the data it receives, switching an array of LEDs on when it sees a 1 and turning it off when it sees a 0.This light is picked up by an array of photodiodes on the receiver. This signal is amplified and filtered to produce a clean signal as similar as possible to what was output by the transmitter MCU. This signal is then sampled by the MCU on the receiver end. Each bit sent by the transmitter is sampled 16 times, and the receiver determines whether it's a 0 or 1 based on whichever bit appears more in that 16-bit section. This data is sent to the computer through a serial connection to be processed by a MATLAB script. The script does the down-sampling and converts the bits into meaningful symbols, either text or audio. At this point, the transmission is complete. However, in their implementation, this success was only apparent on the transmission end of the system. The audio was 
transmitted flawlessly but the receiver was not able to convert the captured signal back into proper form due to problems in the ADC's of the microcontroller. Sampling errors accumulated to the point where they could not be filtered out by the down-sampling error correction, resulting in a meaningless output.

The authors [4] have proposed a number of modulation techniques and thoroughly analyzed in literature for optical wireless communication systems. Each modulation technique has its unique attractive features as well as its challenges. Some are very simple to implement and bandwidth efficient like the On-Off keying (OOK). Pulse position modulation (PPM) provides the unparalleled power efficiency in line of sight (LOS) links but the performance degrades severely in dispersed communication channel.

A prototype was designed to demonstrate wireless VLC using RGB LEDs and sensors[7]. As shown in Fig 2.1, on the left are the RGB LEDs used as signal transmitters. The right side is the RGB sensor, which is used as a receiver. The RGB LEDs enable parallel signal communication, and a PSoC (Programmable System-on-Chip) microcontroller is used to control them, thus significantly reducing the need for extra circuits. Pulse Width Modulation was used to switch RGB LEDs at high speeds. The characteristics of both the variation in color and change in intensity of each RGB LED and RGB sensor were analyzed to realize multiple value signals communication by using RGB color.

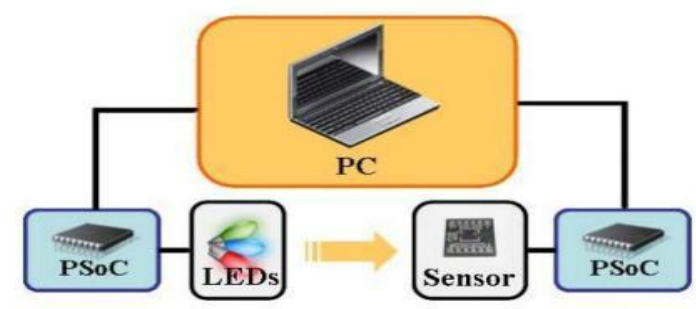

Fig. 3.1 -PSoC (Programmable System-on-Chip) VLC setup

An indoor visible light communication [6] system using white LEDs under consideration. All the lights in the room are replaced by LEDs. The LEDs are not only used for illuminating the room but also for an optical wireless communication system. On-off Keying Return-to-Zero (OOK-RZ) coding is used for modulating white LEDs. Optical lighting and optical transmission of the white LEDs have been tested to evaluate the requirements of using VLC for indoor applications. The effects of the delay problems faced in the high data rate transmission have been studied and presented.

\section{Hardware Implementation:-}

Hardware part of the project includes 2 Arduino boards, Light Emitting Diode (LED), a Photovoltaic cell, few resistors and a 2N2222A transistor. The transmitter consists of an Arduino board and a LED, while the receiver complements it with a Photovoltaic cell and another Arduino board. Further sections explain each of the component used in detail and the problems faced while choosing them for the required specification.

\section{Arduino Board:-}

The board used is Arduino Uno. The Uno is a microcontroller board based on the ATmega328P. It has 14 digital input/output pins (of which 6 can be used as PWM outputs), 6 analog inputs, a $16 \mathrm{MHz}$ quartz crystal, a USB connection, a power jack, an ICSP header and a reset button. It contains everything needed to support the microcontroller; it can be either powered using the USB port which is connected to a Personal Computer or power it with an AC-to-DC adapter or battery.The Uno board is the first in a series of USB Arduino boards.

LED:-

The light is not particularly bright, but in most LEDs it is monochromatic, occurring at a single wavelength. The output from an LED can range from red (at a wavelength of approximately 700 nanometers) to blue-violet (about 400 nanometers). Some LEDs emit infrared (IR) energy (830 nanometers or longer); such a device is known as an infrared-emitting diode (IRED). We decided to choose the standard LED available to us because we could not find the LED of our required specification. 


\section{ANALOG DESIGN OF TRANSMITTER:-}

The purpose of a transmitter is to send data to the receiver so that the other side can process and interpret the data. In our analog design of the transmitter, we used LEDs to transmit light, which would be used to transmit data. The LEDs would modulate on and off to transmit $1 \mathrm{~s}$ and $0 \mathrm{~s}$.

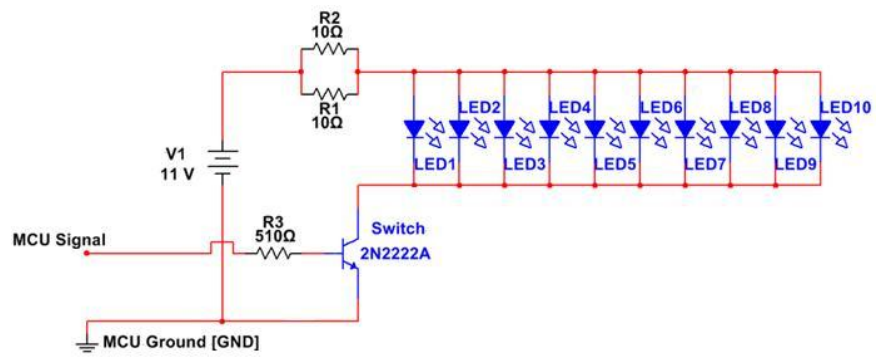

Fig 4.1:- VLC Transmitter Analog Circuit

The source of the data that would be sent to the LEDs would be the ARDUINO of the transmitter circuit. The current from the ARDUINO to the LEDs is not adequate as it was approximately $23 \mathrm{~mA}$ of total current coming from the Arduino to about 10 LEDs directly with zero resistance. This is approximately comes out to around 2-3 mA for each LED in our system which results in little light produced. When the BJT turns on, the collector and emitter connect and the power from the batteries reaches the LEDs. With the 2N2222A supporting high frequencies, It can turn the switch on and off quickly (up to $150 \mathrm{MHz}$ ) so there are no issues with the speed capability. A $510 \Omega$ resistor is used to limit the amount of current travelling from the ARDUINO PWM data pin to the base pin of the BJT. The amount of current flowing is measured to be approximately $4.7 \mathrm{~mA}$. The collector current is approximately $360 \mathrm{~mA}$. The beta of our BJT can be calculated by dividing the collector current by the base current. $\beta$. The beta for our configuration was approximately 76.6. The optimal beta for a 2N2222A transistor is 100 but can largely vary depending on how much base current is flowing as well as the temperature of the transistor.

\section{Photo-Voltaic Cell;-}

The process is both physical and chemical in nature, as the first step involves the photoelectric effect from which a second electrochemical process takes place involving crystallized atoms being ionized in a series, generating an electric current.

\section{Software Implementation:-}

\section{Transmitter:-}

Software implementation of the transmitter is as follows:

* Text of any type i.e. Alphanumerical and Special characters are given as input to Arduino using a computer serially.

* An Array is initialized with Start of Text (STX) as 3 appended in the begging of the array.

* The Arduino waits for a serial input, it will continue further processing only if there is a serial input from the computer.

* When any text is given as input, each character of the text is taken separately.

* Each of the character is converted into its corresponding ASCII value separately.

* The ASCII values are then converted to their Binary equivalent using repetitive division by 2 .

* Converted binary data is then stored into the array with STX as the starting bits.

* End of text (ETX) is appended with the array and then sent to Digital pin no 13 of the Arduino.

* LED is interfaced at port no 13 and corresponding depending on whether the data is 1 or 0 the LED switches on or off respectively i.e. the LED is on-off Keyed with respect to the data. 


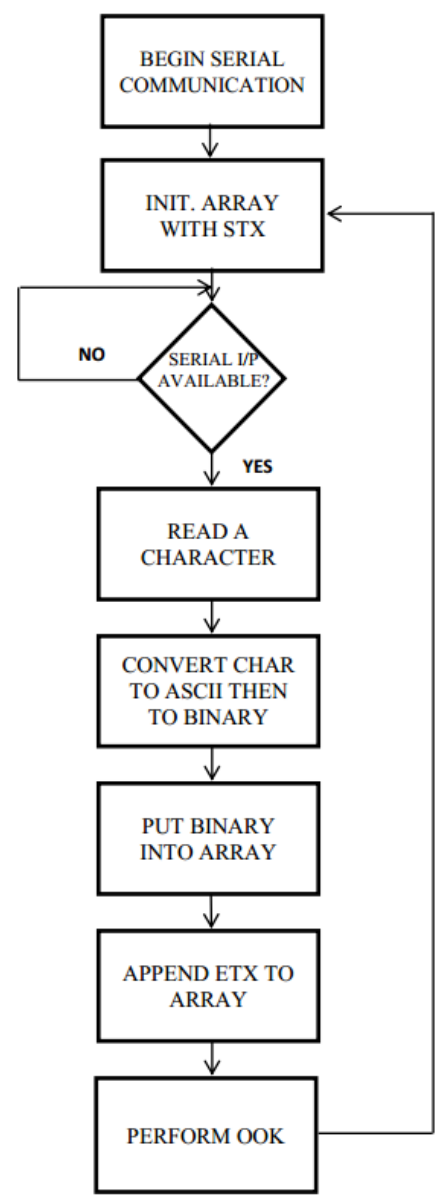

Fig. 5.1:- Transmitter Flowchart

\section{Receiver:-}

Software implementation of receiver is as follows:

* The solar cell is always receiving junk data and decodes it as 0 . The serial data is not recognized as 1 until a certain threshold is crossed.

* Arduino starts receiving data from the transmitter.

* Arduino waits for STX from the transmitter i.e. only if STX is equal to 3 will then be further program executed, else loops back to previous step.

* After the STX is received next 8 bits will contain the binary equivalent of the transmitted character.

- The binary equivalent of the 8 bit data is first converted into its ASCII equivalent. For Example, Consider $01000000.0 * 2^{\wedge} 0=00 * 2 \wedge 1=00 * 2^{\wedge} 2=00 * 2 \wedge 3=00 * 2 \wedge 4=00 * 2 \wedge 5=01 * 2 \wedge 6=640 * 2^{\wedge}$ $7=0$ Decimal $=0+0+0+0+0+0+64+0=64$.

* It waits till ETX is received only then will further processing be done, else it loops back to 2 step and stops reception of further data. If ETX is received then the ASCII code is type casted into the corresponding character and displayed on the monitor faithfully. 


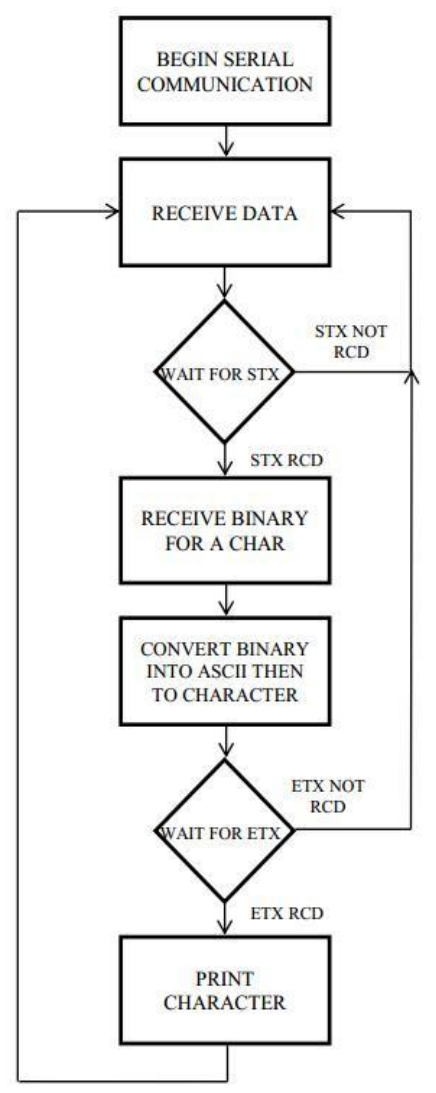

Fig. 5.2 - Receiver Flowchart

\section{Advantages:-}

The bandwidth is much larger than the radio frequency bandwidth, which ranges from $3 \mathrm{kHz}$ to $300 \mathrm{GHz}$. With a larger bandwidth it is possible to accommodate more users and potentially achieve higher transfer rates because each user can be given a larger portion of the bandwidth to transfer information. If the communication system will be used in hospitals, the transmissions will not occur in the Industrial, Scientific, and Medical (ISM) band, therefore not interfering with medical devices. On top of having a higher bandwidth, the frequency spectrum has less regulation than the radio spectrum. With little regulation, the user will be able to choose any frequency to transfer information. If visible light communication systems become more popular, regulations could be placed on these forms of data transmission for the same reasons that they were placed for the radio spectrum. The next major advantage that visible light systems have over other communication systems is its abundance.

Another unique quality of VLC Systems is that by using visual light to transmit data, the data cannot be accessed by any device not in the same room as the system. This unique feature has caused VLC Systems to be of great interest in groups that are concerned about keeping transmissions secure, such as personnel in the defense sector.

Another possible application for a VLC system is indoor geo-location. Currently GPS systems are mostly incapable of functioning within buildings. Since a VLC System consists of a number of different lights, it would be capable of determining the position of devices within a room through triangulation. This could be useful in situations where the location of a person inside a building that has become dangerous in some way needs to be determined.

\section{Disadvantages:-}

While there are a large number of advantages to the use of a VLC system, there are also a number of drawbacks. Of these drawbacks the most obvious is the fact that it will only work in places where there are electronic lights. Conventional Wi-Fi can function a decent distance from a house, allowing internet access while outside. A VLC 
system would not be able to achieve this due to the need for a lighting system. It will generally work best in confined spaces where it is easy to ensure the whole room is being covered in the light.

Another disadvantage to VLC systems is the issue of blocking the light required to receive the signal. It would be very easy to unintentionally block the receiver of the system. This would cause the signal to be interrupted, potentially by things like a person walking through a room. While this disadvantage could be mitigated my using multiple light sources, it would still be very easy to inadvertently interrupt the connection by blocking the light sources.

Another potential problem would be seen mostly with outdoor systems, such as a traffic light system previously described. There could be a number of issues with the system functioning well depending on the weather outside. On cloudy days, it may work while on sunny days it most likely would not. On days when it is raining, snowing, or foggy the signal might be prevented from being transmitted well enough that it could be used. Even indoor systems with many windows might experience this issue.

If the intensity of ambient light is greater than that of the light from our system, the signal-to-noise ratio (SNR) is low, which will distort transmitted data. To compensate for this, the SNR will be maximized by setting thresholds on the microcontroller based on voltage signals produced by the ambient light in conjunction with the transmitter signal. Also, the system will only be maximized when the LEDs are directly facing the sensor. If the angle is changed even slightly, the maximum range of the system will decrease significantly. The easiest solution is to ensure that the transmitter and receiver are facing directly at each other.

\section{Applications:-}

Lights in the visible spectrum are used everywhere, providing several opportunities to apply visible light communications. There are many applications in which data transfer via VLC systems could be useful including traffic lights, which could utilize systems to optimize traffic flow; television sets, which could supply a user with information on current show listings; and hospitals, which could utilize the systems for more secure transfer of data.

\section{TRAFFIC LIGHTS:-}

There are many modern applications that use visible light to portray information. Using a visible communication system in tandem with these devices can increase the devices' functionality. An example of a device that can benefit from a visible communication system is a traffic or stop light. In a busy intersection, traffic lights use visible lighting to maintain the flow of traffic. Because these lights are common in major cities, incorporating some sort of communication system in them to allow our society to stay connected and up to date with all sorts of information improves overall efficiency through multitasking.

\section{TELEVISION APPLICATION:-}

Another piece of modern technology that uses visible light to portray information is a television. Unlike a traffic light, a television contains thousands of pixels that are constantly changing colors to project an image to its viewers. Because there are many individual LEDs in a television, it could be possible to allocate to a few of them the task of transmitting information through a visible light communication system.

\section{HOSPITALS:-}

Hospitals have many reasons to employ wireless technology. Applications of wireless technology in hospitals include updating information by wirelessly maintaining patient records, collecting data as a real-time handheld patient monitor to detect changes in a patient's condition, or even observing medical images via ultrasound.

Accuracy of information via wireless communication is imperative in a hospital setting. In real time applications in which a patient's physiological conditions are monitored, data loss is intolerable with a packet error rate (PER) of less than 10-4.

Security is necessary in order to maintain confidentiality of patient records, and to ensure that only authorized personnel have access to the data being transferred wirelessly. Interference is perhaps the most significant concern in a hospital environment. 


\section{Result and Conclusion:-}

After conducting many trials, our team came up with a model that could transmit text through visible light, at a reasonable bit rate and a reasonable distance.

The model designed by our team, although basic, is found to effectively transmit text at sufficient speeds and for a sufficient distance. The design of the transmitter was pretty straightforward and the logic used by the team is unique to text. The hardware can be improved by obtaining LEDs with better response time. As Arduinos are known to introduce delays in the range of microseconds, bit rates can be improved by just using LEDs which can respond to such fast switching times.

The receiver was experimented with a lot in order to obtain maximum efficiency. Photovoltaic cell was found to be a good alternative for photodiodes as it provided good sensitivity. Again, the datasheets weren't obtained for the photovoltaic cell used, but the sensitivity was found to be good enough to match the response time of the LEDs at the transmitter.

The system designed transmitted text at a data rate of $1 \mathrm{kbps}$ and a distance of $35 \mathrm{~cm}$. The effects of ambient light were eliminated as much as possible in order to make the system suitable for operation in any given environment.

\section{Future scope:-}

The results achieved in this project were severely restricted due to the lack of availability of required hardware. The data rates and the distance can be improved by using more sophisticated hardware and the performance can be pushed to the maximum by knowing the specifications of the hardware being used.

As an extension to this project, error checking and correction can be implemented. We know that information will not be received if there is an obstruction in the line of sight between the transmitter and receiver.

The data transmitted in this project is text only. But with the exact same setup in hardware and relevant changes in code, the data transmitted can be audio, video or any other type of multimedia communication. The main application of this concept being in Light Fidelity $(\mathrm{Li}-\mathrm{Fi})$, most of the research being done is to transmit data packets from the internet through light. This can also be done as a final year project in the future.

\section{Acknowledgment:-}

On successful completion of our project, we would like to place on record our sincere thanks and gratitude to the concerned people, whose suggestions and words of encouragement has been valuable.

\section{References:-}

1. Joseph C. Palais (1988), Fiber Optic Communications second Edition, Second edition. Prentice Hall.

2. Y. Ohno (2004), "Color Rendering and Luminous efficacy of White LED Spectra", Proceedings of IEEE SPIE the International Society for Optical Engineering, Vol. 5530,pp. 88-98 (2004).

3. T. Komiyama, K. Kobayashi, K. Watanabe, T. Ohkubo and Y. Kurihara, "Study of Visible Light Communication System Using RGB LED Lights”, in Proc. IEEE SICE'11, Sept. 2011, Tokya, Japan. pp. 1926 -1928 .

4. Z. Ghassemlooy, Fellow IET, Senior member IEEE, W.O.Popoola, S.Rajbhandari, M.Amiri,"Modulation Techniques for Wireless Infrared Communication" S. Hashemi Optical Communications Research Group, NCRLab., North Umbria University, Newcastle upon Tyne, UK.

5. T. Komiyama, K. Kobayashi, K. Watanabe, T. Ohkubo and Y. Kurihara, "Study of Visible Light Communication System Using RGB LED Lights”, in Proc. IEEE SICE’11, Sept. 2011, Tokya, Japan. pp. 1926 1928.

6. T. Komine and M. Nakagawa, "Fundamental Analysis for Visible-Light Communication System Using LED Lights”, IEEE Transactions on Consumer Electronics, Vol. 50, no. 1, pp. 100-107, February 2004.

7. T. Komiyama, K. Kobayashi, K. Watanabe, T. Ohkubo and Y. Kurihara, "Study of Visible Light Communication System Using RGB LED Lights”, in Proc. IEEE SICE’11, Sept. 2011, Tokya, Japan. pp. 1926 -1928 . 